\title{
Simplified the QoS Factor for the Ad-Hoc Network Using Fuzzy Technique
}

\author{
Dhafer R. Zaghar, Thulfiqar S. Aldeen A. AL Wahab \\ Computer \& Software Engineering Department, College of Engineering, Almostansyriya University, Baghdad, Iraq \\ Email: DRZ_RAW@yahoo.com, Thoalfqar_almashaikhy@yahoo.com
}

Received July 4, 2013; revised August 4, 2013; accepted August 14, 2013

Copyright (C) 2013 Dhafer R. Zaghar, Thulfiqar S. Aldeen A. AL Wahab. This is an open access article distributed under the Creative Commons Attribution License, which permits unrestricted use, distribution, and reproduction in any medium, provided the original work is properly cited.

\begin{abstract}
Quality of Service (QoS) is a tool to measure the efficiency of the Ad-hoc network. QoS is a complex function because it depends mainly on four factors (throughput, packet delivery ratio, end-to-end delay and jitter). These four factors are functions of internal factors and are variables with the time. In the result the QoS is an ambiguous tool. This paper proposes a brand-new method to solve this ambiguity, it will use the fuzzy technique to simplify the QoS factor and summarize it in a simple form or in a single value for each application. So it will summarize all factors of the QoS in a single value for the network if the applications share is defined. This paper will apply the propose method on Mobile Ad-hoc NETwork (MANET) with different protocols. The new algorithm will summarize the efficiency of each protocol in a single (crisp) value for all applications. Finally, an important conclusion is proved, by experimental result, implying that higher throughput does not usually mean high QoS supported by the protocols.
\end{abstract}

Keywords: QoS; Ad-Hoc Routing Protocols; MANET; Fuzzy

\section{Introduction}

MANET is collection of wireless nodes which can connect without the use of any existing network infrastructure (on the fly) to form a wireless network. Each node in MANET can serve as a router to forward packets on the behalf of other nodes and as an end point to the user, this aspect made the router free to move that causes dynamically and unpredictably network topologies change. Topology change and other MANET characteristics like hidden terminal problem, battery and process constrain, link unreliable, intermittent connectivity, limited bandwidth, variable capacity and security made merely traditional routing protocols are not applicable to this type of network and at the same time possess some challenges to the MANET protocols designer. The widespread use of MANET application in battlefield communication, sensor network, emergency and disaster, extends network to the area that needs temporary internet service, vehicular, education and offices network which has demand to achieve QoS assurance in MANET. Different definitions for QoS had been introduced in the literature, the official definition given by regularity bodies, like International Telecommunication Union (ITU), European Telecommunications Standards Institute (ETSI) and Internet En- gineering Task Force (IETF). Intrinsic and perceived QoS must be expressed in term of some parameters that allow the assessment of the provided QoS, a set of parameters for intrinsic QoS that is meaningful for most networks are throughput, packet delivery ratio, delay, jitter [1]. QoS parameters limitation variation depends on the type of application and end users. QoS routing protocols have an essential role in MANET QoS mechanism, since it is their task to find which nodes, if any, can serve application requirements [2]. Huge numbers of works existing in Ad-hoc variation either propose new protocol, algorithm or enhance one of the QoS parameters with the aim to satisfy QoS routing or requirements. Yet, no one of these works had been considered the best due to the MANET characteristics and there's no fundamental criterion for works evaluation with respect to application requirement.

The rest of the paper is organized as follows. In Section 2, a brief reference to related works has been presented. In Section 3, a description for the proposed system with protocols performance is covered. Section 4 simulates the case study with results obtained from simulation. Section 5 summarizes the main conclusion with future work. 


\section{Related Works}

Different simulator where used by the researchers to evaluate the performance of the various Ad-hoc routing protocols. The performance of four different Ad-hoc protocols, Optimized Link State Routing (OLSR), Dynamic Source Routing (DSR), Ad-hoc on Demand Distance Vector (AODV) and Temporary Ordered Routing Algorithm (TORA) were evaluated, in four different scenario situations, and analyzed in [3]. Comparative evaluation within mobile Ad-hoc networks' routing protocols from reactive, proactive and hybrid categories is done in [4] the results showed the lead of Proactive (OLSR) over Reactive (AODV) and hybrid (TORA) protocols in routing traffic for dynamic changing topology. Moreover they have concluded that OLSR is a very effective and efficient route discovery protocol for MANETs. A heuristic and distributed route discovery method named RLGAMAN that supports QoS requirement for MANETs is proposed in [5], this method were based on Reinforcement Learning (RL) and Genetic Algorithm (GA) to find more new feasible paths, comparison with QoSAODV showed the network performance is improved obviously and RLGAMAN is efficient and effective. In [6] OLSR enhanced by Multi-Constrained Path (MCP) QoS Routing, this method based on nonlinear cost function which is the combination of multiple additive QoS parameters, simulation demonstrates the important improvement over the standard protocol. Issues involved with QoS routing is studies in [7], then the authors presents an overview and comparison of existing QoS based revisions done on AODV protocol. In [8] the impact of scalability on three different Ad-hoc (Destination-Sequenced Distance Vector (DSDV), AODV and DSR) are analyzed, the result show that the performance of DSR decrease when the size of network increase and the overall performance of DSDV is less than AODV and DSR whereas AODV is QoS-aware routing protocol under the effect of scalability in terms of variation in number of nodes, mobility rate and packet intervals. The performance of specific network under different Ad-hoc protocol (AODV, TORA, DSR, OLSR) is evaluate in [9], the OLSR outperform other protocols in delay and throughput. Description to the characteristic of Ad-hoc routing protocols (AODV, TORA, OLSR) based on QoS parameters PDR, end to end delay and network load by increase number of nodes in network is given in [10] the comparison result prove that AODV and TORA perform well in dense network than OLSR in term of PDR. In [11] an overview of QoS routing metrics, resources and factors affecting performance of QoS routing protocols, the relative strength, weakness, and applicability of existing QoS routing protocols are also studied and compared. In [12] two Ad-hoc protocol (DSDV and AODV) were examined, from the overall analysis AODV protocol is better than DSDV. In [13] performance comparison of five popular mobile Ad-hoc network routing protocols (AODV, Dynamic MANET On demand (DYMO), OLSR, Zone Routing Protocol (ZRP), DSR) with different pause time is evaluated. In [14] a comparison between number of routing protocols, AODV, DSDV, DSR, Location-Aided Routing (LAR1) and Wireless Routing protocol (WRP), and their performance analysis is based on different network parameters. Qualitative and quantitative analysis of reactive and proactive routing protocol performance evaluation for OLSR, DSDV, AODV and DSR based on metrics such as security, multicasting, packet delivery ratio, average end-to-end delay and routing load is present in [15], the results shows that there is no routing protocol in the current stage without modifications can provide efficient routing to any size of network, regardless of the number of nodes and the network load and mobility. AODV shows better performance than DSDV, OLSR, and DSR. Finally a most recent survey on routing protocols of MANETs by using QoS metrics is covered in [16].

From previous related works, the main conclusions are: the results of the different protocols performance are conflicted, vague (if these protocols satisfy applications and users requirements?). The aim of this paper is to propose a brand new method for Ad-hoc protocols performance evaluation based on applications standards QoS parameters by designing a new system using Hierarchal Fuzzy System (HFS) for protocols evaluation.

\section{Performance Evaluation System}

\subsection{General System Design}

Using fuzzy logic approach for academic performance evaluation is in general fairly new; it has reached a wide range of application areas [17]. According to the tradeoff between parameters, vague of information (i.e. unpredicted mobility of the nodes), applications requirements and NP-problem, QoS in MANET cannot be calculated certainty but there is a range of acceptance to the QoS. To deal with this problem soft computing [18] will be involved; the most important part of soft computing is fuzzy logic. The general structure of the system is shown in Figure 1, where the system has two types of inputs.

1) Parameters input which obtained either from simulators or real networks.

2) Type of applications applied to the network.

The output of the system is a single crisp value represents the performance of Ad-hoc protocol in the network.

\subsection{Protocol Performance}

The QoS evaluation of the Ad-hoc protocol is done by HFS system. The four QoS parameters (throughput, endto-end delay, PDR, jitter) are classify according to its 


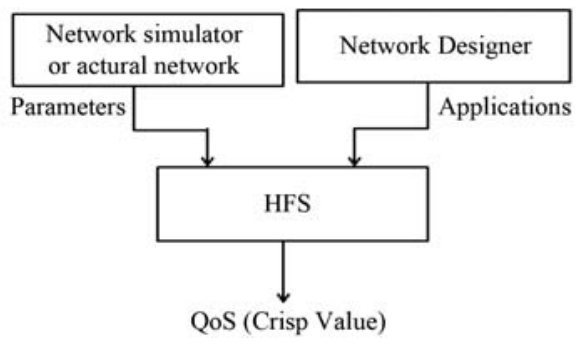

Figure 1. General system structure.

covariant commensurate into two branches. QoS ${ }^{+}$: comprise parameters that have positive effect on the overall QoS. Throughput and PDR are belonging to this branch. As these two parameter increase the QoS support of the Ad-hoc protocol increase, regardless the effect of other parameters. $\mathrm{QoS}^{-}$: comprise parameters that have negative effect on the overall QoS. Jitter and end-to-end delay belonging to this branches. Regardless on the other parameters as these two parameters decrease, the overall QoS of Ad-hoc protocol increase.

Each application has pre-specific limited range of parameters requirements [19-21], QoS parameters that achieved from the Ad-hoc routing protocol within this range or larger made application performance accepted from the end user and simultaneously reflecting the QoS performance of the routing protocol. To get the best result the QoSHFS is formulated from two layers [22] arranged in a hierarchal tree, as shown in Figure 2. Fuzzification, QoS parameters fuzzification based on the limited ranges that given in standards QoS application requirement tables, process is done using triangular and trapezoidal membership function, due to these two membership function advantage in HFS [23]. The left side from Figure 2 (layer 2.1) represent $\mathrm{QoS}^{+}$branch and the right side (layer 2.2) represent $\mathrm{QoS}^{-}$branch. The outputs from layer two $\left(\mathrm{QoS}^{+}\right.$and $\left.\mathrm{QoS}^{-}\right)$use as inputs to the upper layer (layer 1) to evaluate the final performance of any Ad-hoc protocol. The three fuzzy inference systems of QoSHFS are modeled using Mamdani rule (IF-THEN) because; it's transparent and offer interpretability of fuzzy sets and fuzzy rules [24] and Mamdani model allow us to assign weight for each QoS parameter implied by the IF-THEN rule, this aspect is very important in QoSHFS since applications performance is depend largely on specific types of QoS parameters, as example Real Time Application (RTA) is more sensitive to delay whereas Non-RTA is more sensitive to packet loss parameters $[25,26]$ to a certain level.

\section{Case Study and Result}

The software used for designing the QoSHFS is MAT$\mathrm{LAB}^{\circledR}$ version R2010b. To make the QoSHFS very general, network parameters will import to QoSHFS from a

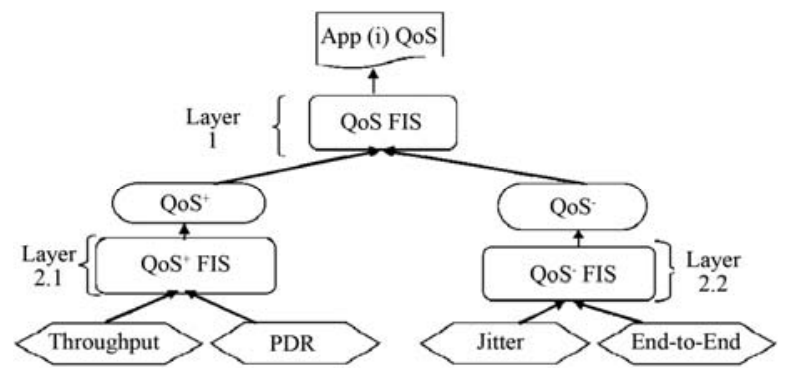

Figure 2. Protocol QoSHFS.

spreadsheet in (.xls) form, this feature enable the network designer to input the parameters obtained from actual network manually to the spreadsheet or automatically from network simulators. The QoS evaluation procedure is shown in Figure 3. In order to investigate the performance of QoSHFS, a network for an office will be simulated as the office employer demands. OPNET Modeler simulator 14.5 is the evaluation technique used in this study [27]. OPNET is one of the most extensively used commercial simulators based on Microsoft Windows platform, which incorporates most of the MANET routing parameters compared to other commercial simulators available [28].

This Office depend mainly on, E-Mail application to serve all employer requirement, E-Mail installed on static and mobile PCs and secondary on voice application, installed on PDA or cell phone, the voice application used by security guards to support connectivity between them, or between them and the mayor cell. The simulation time were set to $1200 \mathrm{sec}$ since simulations need more than $1000 \mathrm{sec}$ of time to reach the steady state [29], and all node where assumed with the same capability supported with high power wireless LAN module [30]. Simulation parameters that will use for different scenarios are listed in Table 1. The most widely and well known Ad-hoc protocol will be apply to this network OLSR, AODV, TORA and DSR in four scenarios where:

- Scenario \#1 OLSR.

- Scenario \#2 AODV.

- Scenario \#3 TORA.

- Scenario\#4 DSR.

The QoS parameters requirements for E-Mail and voice application are listed in [19], the voice application (RTA) is effected directly by the four QoS parameters (Jitter, PDR, throughput and end-to-end delay). E-Mail application (non-RTA) is effected by only two QoS parameters (throughput and end-to-end delay). Jitter does not have any effect on the E-Mail performance (not-applicable) and packet loss ratio must be zero.

Meaning that if there is any packet loss will cause E-Mail transmission fail, therefore these two parameters will be consider as don't care case. The membership functions for voice and E-Mail QoS parameters are 
Table 1. Simulation parameters.

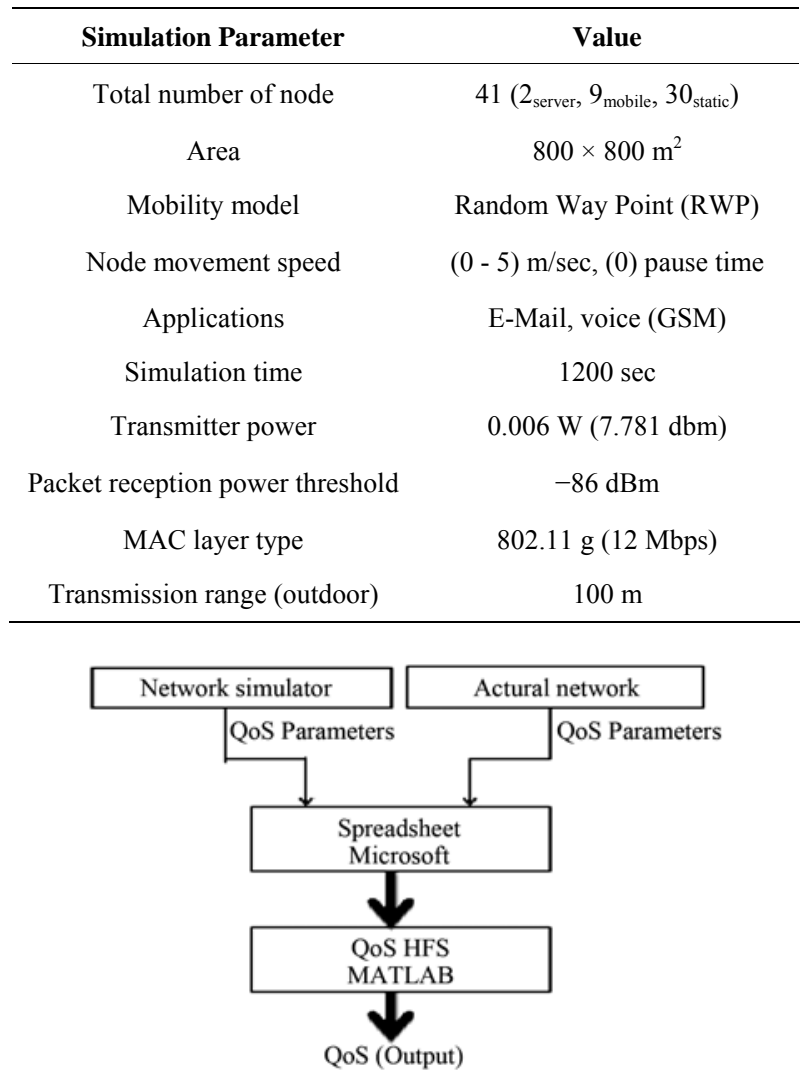

Figure 3. QoS Evaluation steps.

shown in Figures 4 and 5, respectively. Where $X$ axis represents the typical range of QoS parameter requirements and $\mathrm{Y}$ axis represent the parameters membership value that reflects the protocol performance in network. The names and the ranges of the values in these figures are derived from the standard tables of the network specification at the application. The fuzzy conditional rules for each used FIS of the QoSHFS are shown in Figure 6.

The figures in Figure 6 reflect the role of each parameter and its importance in the overall evaluation process, Figure 6(a) show the importance of throughput and PDR on the level of $\mathrm{QoS}^{+}$branch of voice QoS, Figure 6(b) show the effects of jitter and end-to-end delay on the $\mathrm{QoS}^{-}$branch, whereas Figure 6(c) show the final voice QoS as a result of $\mathrm{QoS}^{+}$and $\mathrm{QoS}^{-}$branches output. Figure 6(d) shows the final E-Mail QoS surface reflecting its throughput and response time parameters behavior.

The parameters of intrinsic QoS and their mathematical expression are well defined in the literature, the network results for jitter and end-to-end delay parameters are show in Figures $\mathbf{7}$ and 8, respectively. E-Mail endto-end parameter performances are show in Figure 9.

Traditional results do not give any indication about

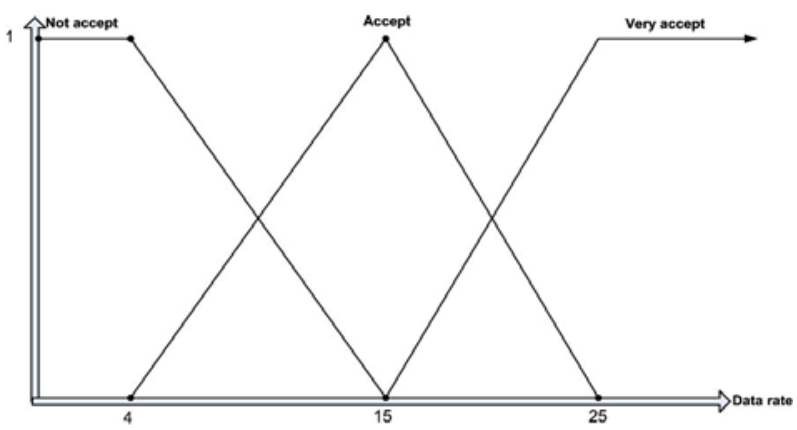

(a)

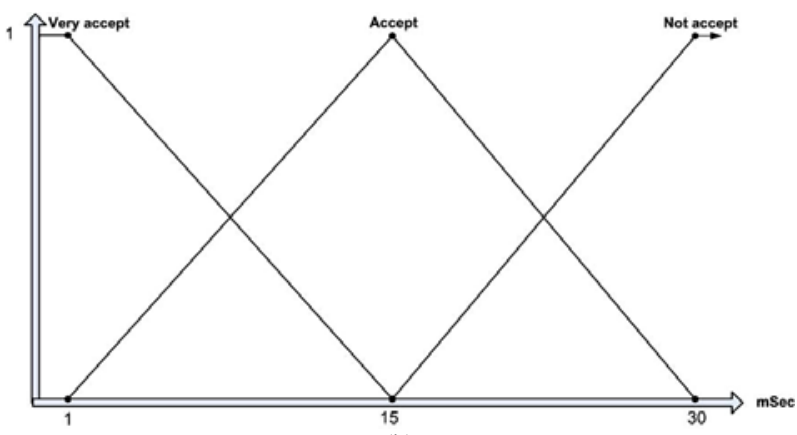

(b)

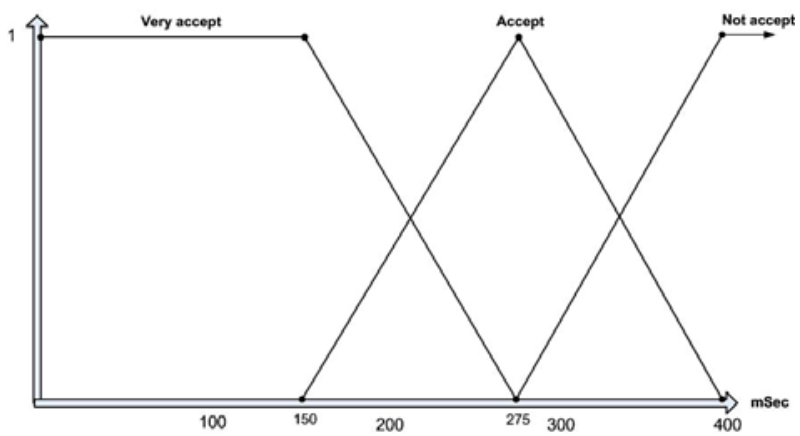

(c)

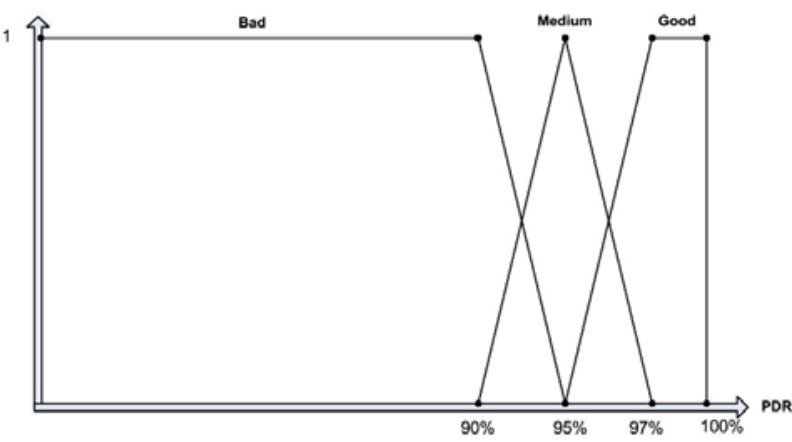

(d)

Figure 4. Voice QoS parameters membership function (a) throughput (b) PDR (c) end-to-end delay (d) jitter.

any of the protocols applied to the network is satisfy applications and users requirements, it just showing that some protocols have low value on some QoS parameters and high on others, but generally there is no clear result about which protocol is more suitable to the network 


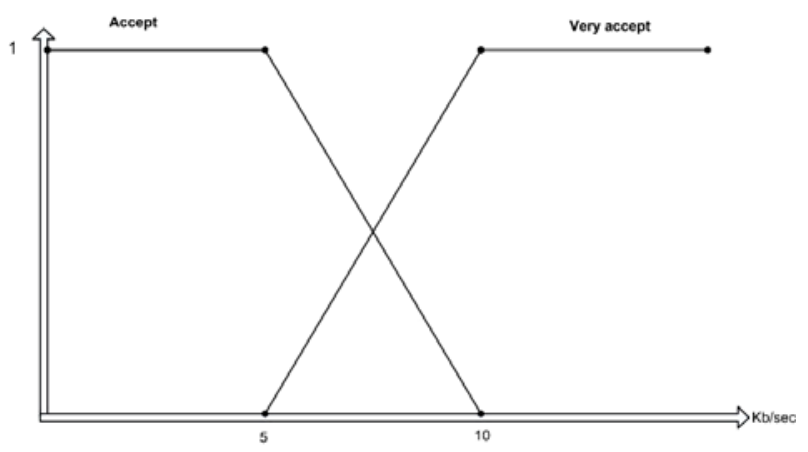

(a)

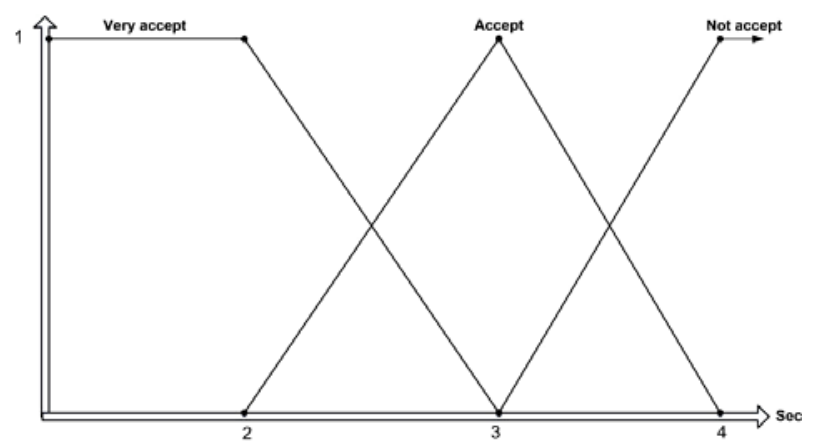

(b)

Figure 5. E-Mail membership functions (a) throughput (b) end-to-end delay.

(Compatible with application parameters limited value). The propose system result, show in Table 2, give a clear analysis about each protocol performance to both E-Mail and voice application.

Each application in network has a weight specified by the network designer, these weights reflect the frequent use of this application from the end users, and in this network it required that E-Mail application have more usage than voice application. Base on this requirement we will assign 0.7 as weight for E-Mail. Since the summation of all applications weights should be 1 then voice application weight will be 0.3 . The final QoS of all the protocol is given in Table 2 by using applications weight in simple additive weight as show in Equation (1). $N$ is the total number of application in deployed in network to the users, $W$ is the weight for each application, specified by the network designer.

$$
\text { QoS protocol }=\sum_{i=1}^{N} W_{(i)} \times \text { QoS } \text { application }_{(i)}
$$

From Table 2, OLSR protocol is the best for this network and second better protocol is DSR whereas AODV and TORA is not suitable for this network. Simulation results base on the worst case where all nodes use the application at the same time (33 node use E-Mail application and 6 node use voice application).

Additionally, when changing the Hello interval of OLSR protocol from the default value $(2 \mathrm{sec})$ to $(4 \mathrm{sec})$

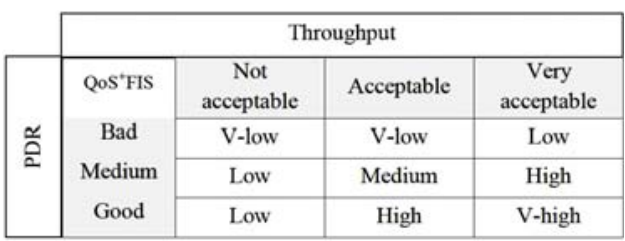

(a)

\begin{tabular}{|c|c|c|c|c|}
\hline & & En & d delay & \\
\hline & QoSFIS & Not accept & accept & Very accept \\
\hline 氮 & $\begin{array}{c}\text { Very } \\
\text { accept }\end{array}$ & Medium & V-high & V-high \\
\hline & accept & Low & High & High \\
\hline & $\begin{array}{c}\text { Not } \\
\text { accept }\end{array}$ & V-low & V-low & V-low \\
\hline
\end{tabular}

(b)

\begin{tabular}{|c|c|c|c|c|}
\cline { 2 - 5 } \multicolumn{1}{c|}{} & \multicolumn{4}{c|}{ 1-QoS } \\
\hline \multirow{4}{*}{ \&oSFIS } & Low & Bad & Medium & Good \\
\cline { 3 - 5 } & V-low & Low & Medium \\
\cline { 3 - 5 } & Medium & Low & Medium & High \\
\cline { 2 - 5 } & High & Medium & High & V-high \\
\hline
\end{tabular}

(c)

\begin{tabular}{|c|c|c|c|c|}
\cline { 4 - 6 } \multicolumn{1}{c|}{} & \multicolumn{5}{c|}{ QoS (End to end delay) } \\
\hline \multirow{2}{*}{$\begin{array}{c}\text { is } \\
\text { Q }\end{array}$} & AoSFIS & Not accept & Accept & Very accept \\
\cline { 3 - 6 } & Normal & Fast & Very fast \\
\cline { 3 - 6 } & Very accept & Normal & Fast & Very fast \\
\hline
\end{tabular}

(d)

Figure 6. (a) Fuzzy matrix rule for throughput and PDR; (b) fuzzy matrix rule for jitter and end-to-end; (c) fuzzy matrix rule for voice application; (d) fuzzy matrix rule for E-Mail application.

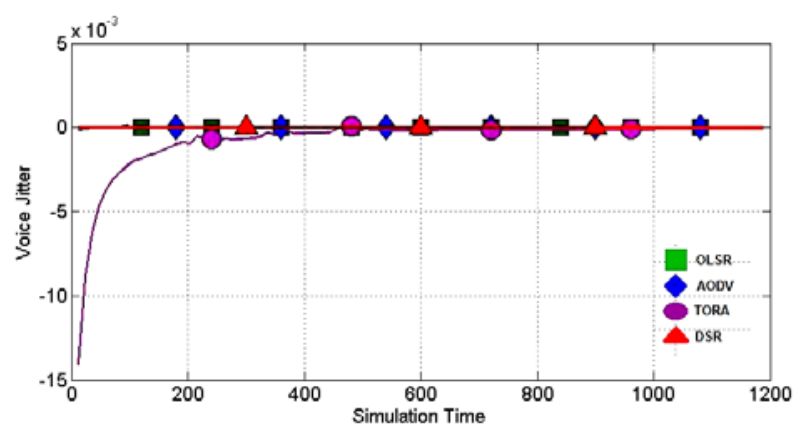

Figure 7. Voice application jitter.

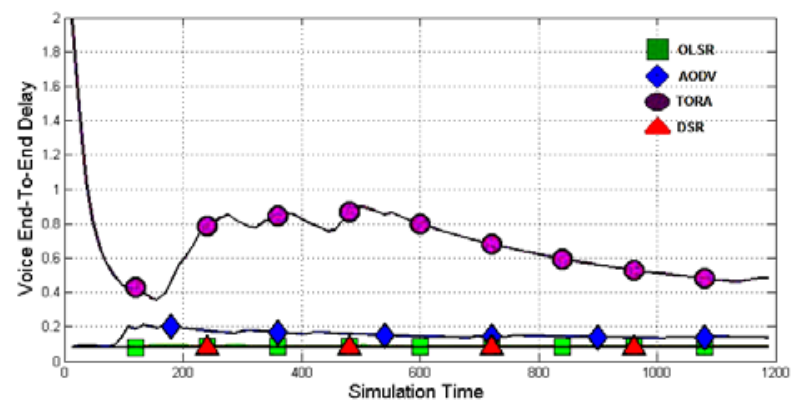

Figure 8. Voice application end-to-end delay. 
Table 2. QoSHFS results for the four protocols.

\begin{tabular}{cccccc}
\hline \multicolumn{2}{c}{ Protocol } & QoS $^{+}{ }_{\text {(voice) }}$ QoS $_{\text {(voice) }}^{-}$ & $\begin{array}{c}\text { Voice } \\
\text { QoS }\end{array}$ & $\begin{array}{c}\text { E-Mail } \\
\text { QoS }\end{array}$ & $\begin{array}{c}\text { Final protocol } \\
\text { QoS }\end{array}$ \\
\hline OLSR & 0.9362 & 0.92 & 0.9367 & 0.8336 & 0.8645 \\
AODV & 0.4910 & 0.92 & 0.6877 & 0.1603 & 0.3185 \\
TORA & 0.4564 & 0.50 & 0.4518 & 0.1603 & 0.2477 \\
DSR & 0.9362 & 0.92 & 0.9367 & 0.3385 & 0.5180 \\
\hline
\end{tabular}

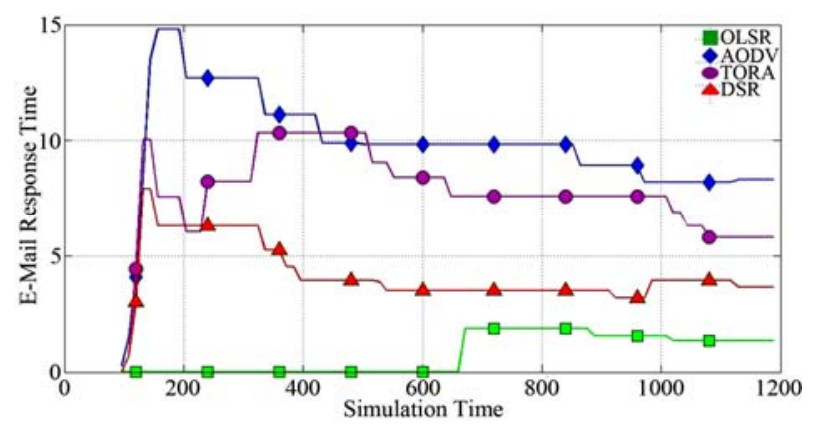

Figure 9. E-Mail application end-to-end delay.

and another time to $(1 \mathrm{sec})$. The OLSR performance from QoSHFS is still (0.8645). Throughput of the different interval is show in Figure $\mathbf{1 0 .}$

Hello interval has large effect on OLSR protocol throughput value but with same protocol QoS performance. When Hello interval is $1 \mathrm{sec}$, the throughput value is very high. When Hello interval changed to $4 \mathrm{sec}$ throughput value become less than the default OLSR. OLSR with these different Hello interval produce same QoS value but with different throughput and as a consequence different channel usage and capacity.

\section{Conclusions and Future Work}

A brand-new system for MANET protocols performance evaluation based on the applications and users' requirements is proposed in this paper. The output of the present system, the single crisp value, the level of the protocols QoS are offered to the end users. System output 1 represents full QoS whereas 0 means there is no QoS or even no network.

The result analysis behavior of the traditional approach and the results of the proposed system are also compared through a case study. The traditional approach results are represented usually by QoS parameters curves, these curves show that the performance of some protocols is high for some parameters and low for others, which are controlled by the protocols algorithm limitation and their type. These curves don't give a clear view about the overall protocols performance for the application in the network. As examples of Figures $\mathbf{7}$ and $\mathbf{8}$ show different parameters curves for each protocol but due to the voice application's requirement all these parameters values are

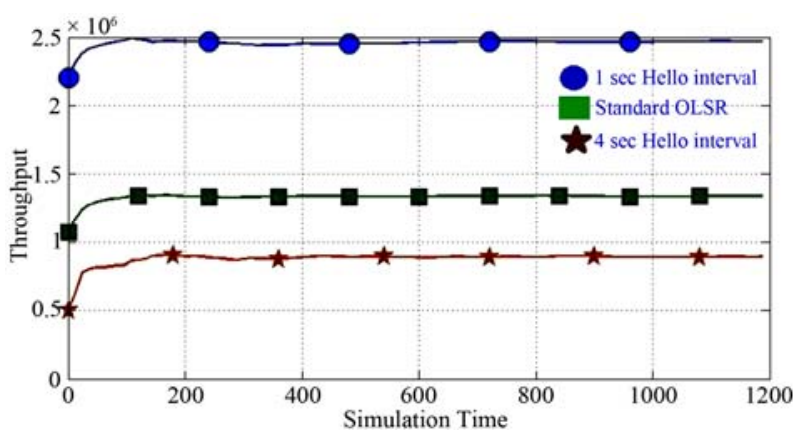

Figure 10. OLSR throughput with different Hello interval.

in the accepted parameters ranges, which meaning no protocol outperforms the other in these two parameters. The proposed system (QoSHFS) results show the OLSR (proactive) performance is the best for this type of application QoS requirements in this network environment because the system output for OLSR is (0.8645). DSR (reactive) protocol has QoS (0.5180). AODV (reactive) and TORA (hybrid) are not applicable in this type of network because their QoS are (0.3185) and (0.2477), respectively. DSR is also a reactive protocol but it typically carries complete route information, if the packet header overhead increases, the performance of DSR will decrease. Throughput is the foregone conclusion of the other parameters performance (PDR, overhead, etc.) which means that when throughput in some protocols is high, it does not absolutely mean the performance of that protocol is good or satisfies users and applications' requirements.

On the other hand, in the future, the development of the proposed system includes all applications that may deploy in MANET (HTTP, video conference, FTP, etc.) using the same method that is proposed in this paper, beneficiary from standard QoS parameters for applications. And proposing a new system for MANET environment evaluation (mobility prediction, type of nodes role in the network, etc.) using Fuzzy Multiple Attribute Decision Maker (FMADM) considering MANET environment is a fuzzy environment affected by the number and distribution of nodes in MANET and nodes speed, velocity and acceleration to be a complete standard official system for the overall performance evaluation of the existing or new Ad-hoc protocols.

\section{REFERENCES}

[1] J. Olmos, R. Ferrus, O. Sallent, J. Perez-Romero and F. Casadevall, "QoS Architecture and Functionalities: AROMA's Perspective," 3rd Edition, Workshop Trends in Radio Resource Management, Barcelona, 21 November 2007.

[2] X. Wang, "Mobile Ad-Hoc Networks: Protocol Design," InTech, Rijeka, 2011. doi:10.5772/548

[3] A. Zaballos, A. Vallejo, G. Corral and J. Abella, “Ad-Hoc 
Routing Performance Study Using OPNET Modeler," Barcelona, 2006.

[4] N. Qasim, F. Said and H. Aghvami, "Mobile Ad Hoc Networking Protocols' Evaluation through Simulation for Quality of Service," IAENG International Journal of Computer Science, Vol. 36, No. 1, 2009, pp. 76-84.

[5] J. Mungara, S. P. Setti and G. Vasanth, "Design and a New Method of Quality of Service in Mobile Ad-Hoc Network (Manet)," European Journal of Scientific Research, Vol. 34, No. 1, 2009, pp. 141-149.

[6] K. Kunavut and T. Sanguankotchakorn, "Multi-Constrained Path (MCP) QoS Routing in OLSR Based on Multiple Additive QoS Metrics," 2010 International Symposium on Communications and Information Technologies (ISCIT), Tokyo, 26-29 October 2010, pp. 226231.

[7] S. Sridhar and R. Baskaran, "A Survey on QoS Based Routing Protocols for MANET," International Journal of Computer Applications, Vol. 8, No. 3, 2010, pp. 15-22.

[8] R. K. Gujral and M. Singh, "Analyzing the Impact of Scalability on QoS-Aware Routing for MANETs," IJCSI International Journal of Computer Science Issues, Vol. 8, Issue 3, No. 1, 2011, pp. 487-495.

[9] R. Al-Ani, "Simulation and Performance Analysis Evaluation for Variant MANET Routing Protocols," International Journal of Advancements in Computing Technology, Vol. 3, No. 1, 2011, pp. 1-12.

[10] P. Kuppusamy, K. Thirunavukkarasu and B. Kalaavathi, "A Study and Comparison of OLSR, AODV and TORA Routing Protocols in Ad Hoc Networks," 2011 3rd International Conference on Electronics Computer Technology (ICECT), Kanyakumari, 8-10 April 2011, pp. 143147.

[11] S. Gangwar, S. Pal and K. Kumar, "Mobile Ad Hoc Networks: A Comparative Study of QoS Routing Protocols," IJCSET, Vol. 2, No. 1, 2012, pp. 771-775.

[12] B. N. Jagdale, P. Patil, P. Lahane and D. Javale, "Analysis and Comparison of Distance Vector, DSDV and AODV Protocol of MANET," International Journal of Distributed and Parallel Systems (IJDPS) Vol. 3, No. 2, 2012, pp. 121-131.

[13] S. Kumar and J. Kumar, "Comparative Performance Analysis of Routing Protocols in MANET using Varying Pause Time," International Journal of Computer Applications, Vol. 47, No. 12, 2012, pp. 22-27.

[14] V. Gupta, "Comparative Performance Analysis of AODV, DSR, DSDV, LAR1 and WRP Routing Protocols in MANET Using GloMoSim 2.0.3 Simulator," International Journal of Computer Applications, Vol. 52, No. 20, 2012, pp. 16-24.

[15] V. P. Patil, "Reactive and Proactive Routing Protocol Performance Evaluation for Qualitative and Quantitative Analysis in Mobile Ad Hoc Network," International Journal of Scientific and Research Publications, Vol. 2,
No. 9, 2012, pp. 1-8.

[16] I. Panda, "A Survey on Routing Protocols of MANETs by Using QoS Metrics," International Journal of Advanced Research in Computer Science and Software Engineering, Vol 2, No. 10, 2012, pp. 120-129.

[17] R. S. Yadav and V. P. Singh, "Modeling Academic Performance Evaluation Using Soft Computing Techniques: A Fuzzy Logic Approach," International Journal on Computer Science and Engineering (IJCSE), Vol. 3, No. 2, 2011, pp. 676-680.

[18] H.-J. Zimmermann, "Fuzzy Set Theory," John Wiley \& Sons, Inc., Vol. 2, 2010.

[19] "3rd Generation Partnership Project; Technical Specification Group Services and System Aspects Service aspects; Services and Service Capabilities (Release 8), 3GPP TS 22.105 V8.4.0," 2007.

[20] J. I. M. Novella and F. J. G. Castaño, "Qos Requirements for Multimedia Services," In: Resource Management in Satellite Networks, Springer Science + Business Media, LLC, 2007, pp. 67-94.

[21] “ITU Recommendation G. 114," 2003.

[22] J. Zajaczkowski and B. Verma, "Selection and Impact of Different Topologies in Multi-Layered Hierarchical Fuzzy Systems," Springer Science + Business Media, LLC, 2011.

[23] M. Centile, "Development of Hierarchical Fuzzy Model for the Evaluation of Inherent Saftey," PHD Thesis, Texas A\&M University, College Station, 2004.

[24] R. Babuška, "Fuzzy Systems, Modeling and Identification," 1997.

[25] K. Farkas, D. Budke, B. Plattner, O. Wellnitz and L. Wolf, "QoS Extensions to Mobile Ad Hoc Routing Supporting Real-Time Applications," IEEE International Conference on Computer Systems and Applications, 8 March 2006, pp. 54-61.

[26] R. Braden, D. Clark and S. Shenker, "Integrated Services in the Internet Architecture: An Overview," Request for Comments (RFC), Network Working Group, 1994.

[27] OPNET, Inc., “OPNET Modeler 14.5.” www.opnet.com

[28] P. Singh, E. Barkhodia and G. K. Walia, "Evaluation of Various Traffic load in MANET with DSR Routing Protocol through Us of OPNET Simulator," International Journal of Distributed and Parallel Systems (IJDPS) Vol. 3, No. 3, 2012, pp. 75-83.

[29] M. Amnai, Y. Fakhri and J. Abouchabaka, "Qos Routing and Performance Evaluation for Mobile Ad Hoc Networks Using Olsr Protocol," International Journal of Ad Hoc, Sensor \& Ubiquitous Computing (IJASUC), Vol. 2, No. 2, 2011, pp. 12-23. doi:10.5121/ijasuc.2011.2202

[30] XG-622H mPCI WLAN Module, Data Sheet, Zcomax Technologies, 2007. 